\title{
Comparative study on earthquake and ground based transmitter induced radiation belt electron precipitation at middle latitudes
}

\author{
N. F. Sidiropoulos ${ }^{1,2}$, G. Anagnostopoulos ${ }^{1}$, and V. Rigas $^{1}$ \\ ${ }^{1}$ Dept. of Electrical and Computer Engineering, Democritus University of Thrace, Kimmeria, 67100 Xanthi, Greece \\ ${ }^{2}$ Institute for Language and Speech Processing/“Athena” R. C., Vas. Sofias 8 Str. 67100, Xanthi, Greece
}

Received: 15 November 2010 - Revised: 19 March 2011 - Accepted: 29 April 2011 - Published: 8 July 2011

\begin{abstract}
We examined (peak-to-background flux ratio $p / b>20$ ) energetic electron bursts in the presence of VLF activity, as observed from the DEMETER satellite at low altitudes $(\sim 700 \mathrm{~km})$. Our statistical analysis of measurements during two 6-month periods suggests that: (a) the powerful transmitter NWC causes the strongest effects on the inner radiation belts in comparison with other ground-based VLF transmitters, (b) the NWC transmitter was responsible for only $\sim 1.5 \%$ of total electron bursts examined during the 6month period (1 July 2008 to 31 December 2008), (c) VLF transmitter-related electron bursts are accompanied by the presence of a narrow band emission centered at the radiating frequency emission, whereas the earthquake-related electron bursts are accompanied by the presence of broadband emissions from a few $\mathrm{kHz}$ to $>20 \mathrm{KHz}$, (d) daytime events are less preferable than nighttime events, but this asymmetry was found to be less evident when the powerful transmitter NWC was turned off and (d) seismic activity most probably dominated the electromagnetic interactions producing the electron precipitation at middle latitudes. The results of this study support the proposal that the detection of radiation belt electron precipitation, besides other kinds of studies, is a useful tool for earthquake prediction research.
\end{abstract}

\section{Introduction}

Energetic charged particle precipitation from the radiation belts has been observed in the ionosphere at middle and low geographic latitudes and has been studied with a series of different methods in the last decades (Paulikas, 1975; Inan et al.,1978; Vampola and Gorny, 1983; Abel and Thorne, 1998; Koskinen, 2005; Katoh et al., 2005).

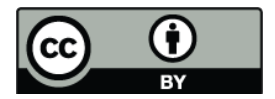

Correspondence to: N. F. Sidiropoulos (nsidir@ee.duth.gr)
The electron precipitation in the environment of the Earth's surface $(\sim 100-2000 \mathrm{~km})$ has been attributed to (a) intense VLF emissions by man-made transmitters (Gamble et al., 2008; Sauvaud et al., 2008; Graf et al., 2009), (b) seismic activity (Galper et al., 1983; Ginzburg et al., 1994; Sgrigna et al., 2005; Fidani and Battiston, 2008; Aleksadrin et al., 2003; Anagnostopoulos et al., 2010a; Sidiropoulos et al., 2010), and (c) lighting during thunderstorms (McCormick et al., 2002). Electron precipitation is also known to follow geomagnetic storms, but this a more global phenomenon.

Space observations have advanced the earthquake precursory signals research on plasma and electromagnetic phenomena in the ionosphere (Boskova et al., 1994; Rothkaehl et al., 2006; Zhang et al., 2010; Athanasiou et al., 2011). Energetic electron precipitation is another tool for earthquake prediction research that has recently attracted increasing interest.

Because of historical perceptions, energetic electron precipitation has been hypothesized to originate mostly from man-made VLF transmitter radiation, although a nonobservational basis has been known to confirm this impression as far as we know. On these lines, Sauvaud et al. (2008), on the one hand noted that theoretical calculations have led to the "rather surprising conclusions" that man-made VLF transmissions may dominate losses in the inner radiation belts (Abel and Thorne, 1998) but on the other hand, their study based on DEMETER electron observations did not supported such a conclusion. Sauvaud et al. (2008) only came to the conclusion that an energetic electron structure associated with the most powerful transmitter of the US Navy in Western Australia extends in latitudes eastward of the west coast of Australia.

Anagnostopoulos et al. (2010a) analyzed DEMETER in detail and described the behavior of energetic electron precipitation before great $(M>6.7)$ earthquakes (EQs). They examined the earthquake occurring in Japan on 16 August 2005, which was selected as a clear representative example

Published by Copernicus Publications on behalf of the European Geosciences Union. 
because of the absence of other great earthquakes in the previous time period (of $\sim 15$ days). The most important finding of this study is a characteristic pattern of the temporal evolution of energetic electron precipitation (EEP) with an intensification at the first phase and a reduction of its strength in the second phase, which reaches a minimum around the earthquake occurrence time (MaxMin model). A spatial-temporal analysis has shown that the EEP almost ceases above the EQ epicenter a couple of (or a few) hours before earthquake occurrence. A "silence" in VLF emission a few hours before earthquake occurrence has also been reported in the ionosphere (Nemec et al., 2009) and on the Earth's surface (Contoyiannis et al., 2005) and it seems that these effects are selfconsistent. Furthermore, a similar minimum before earthquakes has been reported in several other physical parameters, the polarization of the magnetic field (Hayakawa et al., 1996), the geomagnetic activity as inferred from Kp values (Anagnostopoulos et al., 2010c), etc. The MaxMin model of electron precipitation was checked and confirmed for a statistical sample of great earthquakes in Japan, but it is probably a rather general feature of great earthquakes (Anagnostopoulos et al. (2010a, b).

Another interesting result of the study by Anagnostopoulos et al. (2010a, b) is that the earthquake examined seems to control the electron distribution at large distances from the epicenter for several days before its occurrence.

The relative contribution of anthropogenic and earthquake-related belt electron precipitation has recently been addressed and estimated by Sidiropoulos et al. (2010). In this study we have presented results from a statistical analysis of intense energetic electron bursts (peak-to-background flux ratio $p / b>20$ ) in the presence of VLF activity and we have found that other sources (no VLF transmitters) are responsible for $>\sim 85 \%$ of a total of 757 intense EBs examined in two $\sim 6$ month periods. Sidiropoulos et al. (2010) inferred that seismic activity most probably dominates the electromagnetic interactions producing the electron precipitation at middle latitudes.

The purpose of this paper is to extend and further check the results of the study by Sidiropoulos et al. (2010). In the present paper, particular emphasis is given to the distinction of the origin of EEP events by comparing the EBs with the dynamic electric field spectra in the VLF band. Our analysis reveals that the EQ-related EBs are associated with broad band VLF activity, whereas the transmitter-related EBs, as expected, are accompanied by a narrow band spectrum at the transmitter-emitted frequency. In some EBs, both earthquake and transmitter emission seems to influence the radiation belts, but the transmitter-caused effect strongly decreases with distance from the transmitter location. Our results further support our previous conclusion that seismic activity dominates the electromagnetic interactions producing the radiation belt electron precipitation at middle latitudes.

\section{Instrumentation}

DEMETER (Detection of Electro-Magnetic Emissions Transmitted from Earthquake Regions) is a sun-synchronous, low latitude $(\sim 710 \mathrm{~km})$ satellite in a circular, almost polar, orbit (inclination $98.3^{\circ}$ ). Its scientific payload is composed of several types of sensors measuring waves and particles. In this study we use measurements from the two experiments: IDP (Instrument for the Detection of Particles Electrique) (Sauvaud et al., 2006) and ICE (Instrument Champ Electrique) (Berthelier et al., 2006).

The IDP electron spectrometer covers all energies from $0.07-2.5 \mathrm{MeV}$ in 256 energy bands, but our present work is based on the analysis of electron fluxes in the energy bands: $72-526 \mathrm{keV}$ (Band 1), 526-971 keV (Band 2) and 971-2350 keV (Band 3). IDP has a maximum geometrical factor of $1.2 \mathrm{~cm}^{2} \mathrm{sr}$ and it measures electrons in the drift loss cone.

ICE measures electromagnetic and/or electrostatic waves in a wide frequency range, from $\mathrm{DC}$ to $3.175 \mathrm{MHz}$, subdivided into four frequency channels DC/ULF, ELF, VLF and HF. Here we use measurements from the VLF $(0-20 \mathrm{kHz})$ channel.

The EEP events analyzed in the present paper are of the kind observed by DEMETER near the US Naval transmitter, North West Cape, Western Australia $\left(21.81^{\circ} \mathrm{S}, 114.16^{\circ} \mathrm{E}\right)$, also known with the call sign "NWC". The NWC transmitter serves as a radio beacon for submarines of the US Navy and it radiates at a frequency of $19.8 \mathrm{kHz}$ with a power of $1 \mathrm{MW}$. These characteristics have established the NWC transmitter as the strongest VFL transmitter in the world. The NWC VLF transmitter provides a great opportunity for a study on the comparative contribution of the natural and the manmade radiation belt electron precipitation because of its position in geographic latitude. The latitude of the NWC transmitter corresponds to an $\mathrm{L}$ of 1.45 , where a good influence upon $>100 \mathrm{keV}$ electrons in the inner radiation belt is predicted (Sauvaud et al., 2008). Thus a comparative study has been attempted and the contribution of NWC-related EEP bursts and of similar earthquake-related electron bursts (EBs) has been estimated.

\section{Main types of energetic electron precipitation events observed by DEMETER}

In the present study, we have attempted to study the main types of radiation belt electron precipitation events and the corresponding relative contribution of the causes producing each type. For this reason, we first compare the electron precipitation events with various forms of dynamic electric field spectra in the VLF band.

In Fig. 1, on the left of the top panel, we present the fluxtime profiles of DEMETER/IDP energetic electrons in three energy bands (the low energy Band 1, the middle energy 

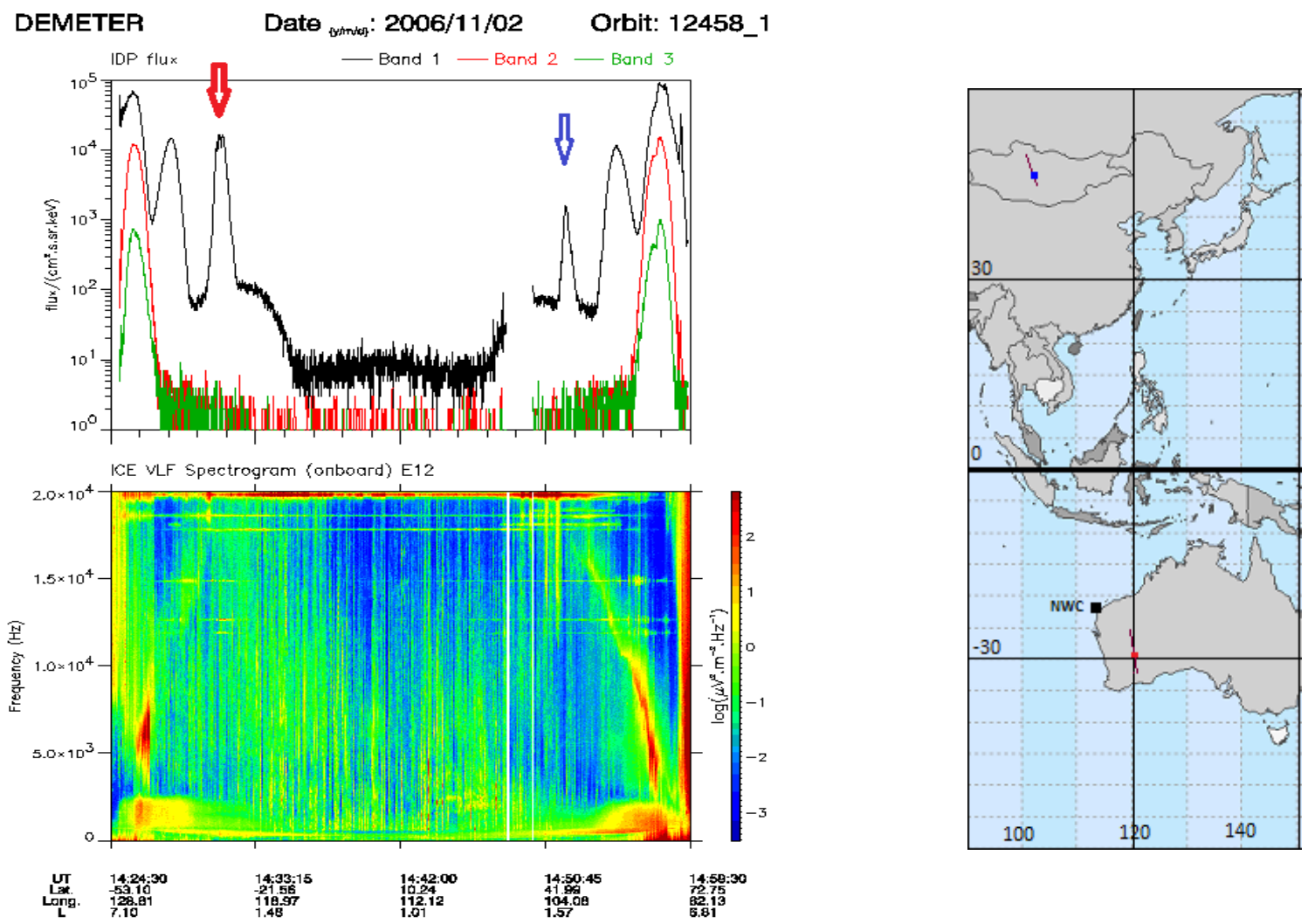

a)

b)

Fig. 1. Example of an electron burst (indicated by the red arrow) related to the NWC transmitter recorded during DEMETER's anodic orbits on 2 November 2006. The upper panel on the left presents the differential intensities in three energy bands (see in the text) as recorded by the IDP experiment, while the lower panel presents the VLF spectrogram from the ICE experiment for the same time interval. The short brown lines and the small red and blue squares on the map on the right side of the figure show the parts of the DEMETER orbit during the NWC related electron burst, its conjugate at North, and the maximum flux in the two cases were observed. No other VLF signal is seen related to the NWC related electron burst except the corresponding to the emitted frequency (red line at the $19.8 \mathrm{kHz}$ ).

Band 2 and the high energy Band 3), for the time interval 14:24:30-14:59:30 UT, 2 November 2006 (anodic, nightside half orbit \# 12458_1). In the bottom panel, on the left, the DEMETER/ICE VLF $(0-20 \mathrm{kHz})$ spectrogram is shown, where the electric field density is inferred by a comparison with the color bar at the right. Below the bottom panel, the Universal Time, the Latitude and the Longitude, as well as the $\mathrm{L}$ shell at the position of DEMETER are noted.

The flux peak at $\sim 14: 31$ UT (indicated by a red arrow) superimposed on the background of the intensity profile of electron Band 1 is a temporal effect, a so-called "anomalous electron burst", not consistent with the shape of the normal flux-time profile at those latitudes $\left(\sim 30^{\circ} \mathrm{S}\right)$. This EB was detected when DEMETER passed very close to the position of the NWC transmitter. The brown line, the red circle, and the black square on the map on the right of Fig. 1 indicate the segment of the DEMETER trajectory during the time of the anomalous electron burst, the point of the trajectory when the flux was observed, and the position of the NWC trans- mitter in West Australia, respectively. We can see that the anomalous electron burst was quite strong with a peak-tobackground flux ratio evaluated as large as $R_{p / b}>10^{2}$. (for a definition of $R_{p / b}$ see Appendix A)

The blue arrow above a peak at $\sim 14: 51: 40$ UT and the blue square over Mongolia on the map indicate the time when the conjugate electron enhancement was detected during the same DEMETER semi-orbit. The conjugate EB shows a flux $R_{p / b}$, about an order of magnitude lower than the value observed on the southern part of the spacecraft trajectory.

What is very important in the present study is the distinction between various types of electric field spectrum observed along with the EBs. As we have already mentioned, it is rather generally accepted that electron precipitation at middle latitudes is mostly due to earth-based VLF transmitters. Indeed, this is the case of the EBs shown in Fig. 1. As we see, the only important VLF activity outside the radiation belts is a narrow band emission (red "line" at the top of spectrogram) centred at the radiating frequency $(\sim 19.8 \mathrm{kHz})$ 

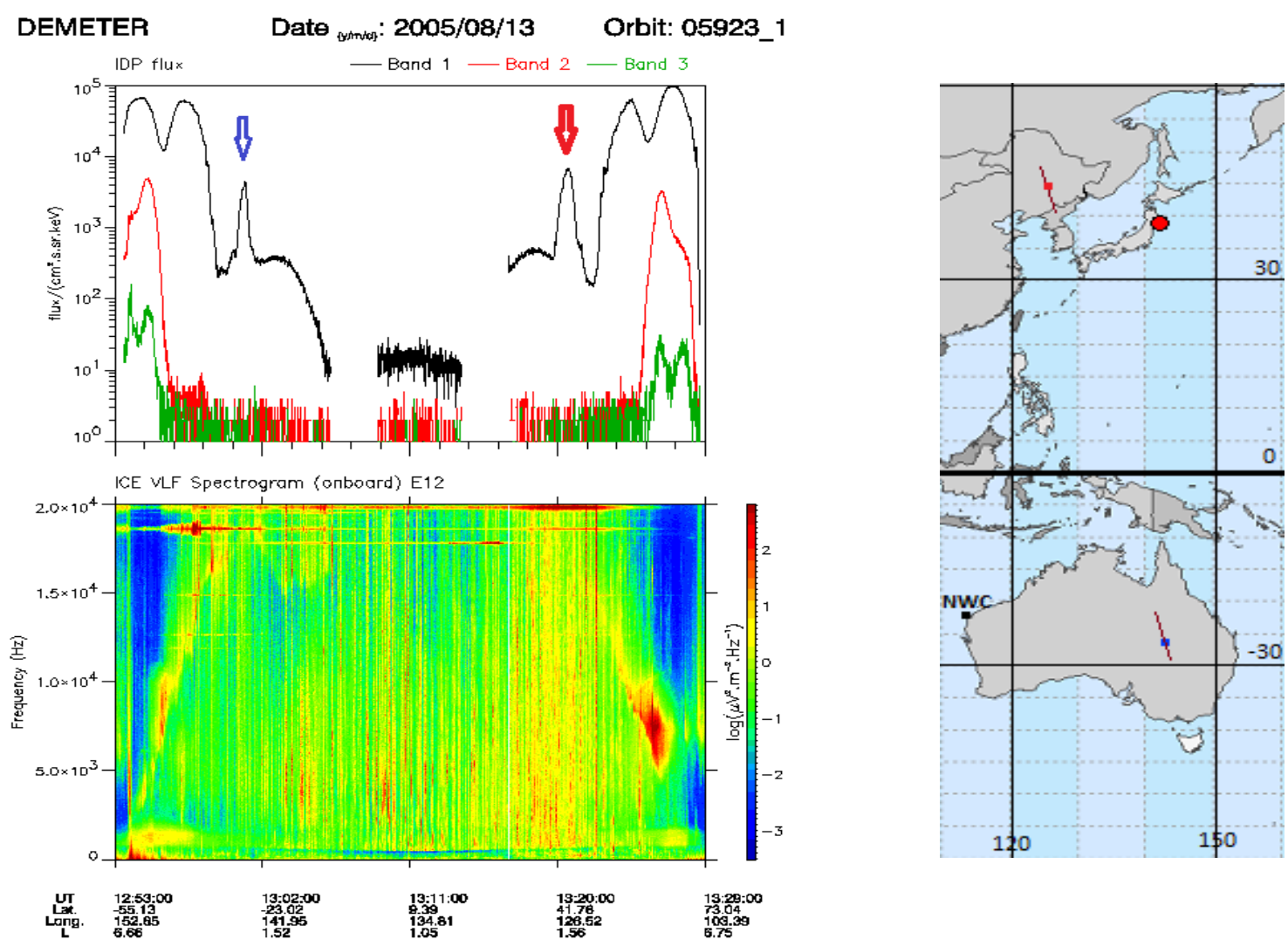

a)

b)

Fig. 2. The figure has been constructed in the same format as Fig. 1. This is an example of an electron burst (red arrow) that is a precursor of the earthquake occurring on 16 August 2005, in Japan (the epicenter marked with a red circle on the map on the right side of the figure). This earthquake-related electron event was accompanied by a broad band VLF signal extending in frequencies all over the scale of the panel ranging from 0 to $20 \mathrm{kHz}$. The narrow band signal in the spectrogram at the top of the $(19.8 \mathrm{kHz})$ figure indicates the NWC transmitter influence.

of the NWC transmitter; there is no other significant VLF emission signal in the spectrogram related to the time of the EB observed by DEMETER near the NWC transmitter of the US Navy. Therefore, we identify this type of electric field spectrogram as representative one of the cases of energetic electron precipitation events triggered by the emissions of earth based VLF transmitters, and particularly of the powered NWC transmitter.

Figure 2 has been constructed in the same format as Fig. 1, but for a semi-orbit during the time interval 12:53-13:29 UT on 13 August 2005, when DEMETER crossed a region from $152.85^{\circ} \mathrm{E}$ to $103.39^{\circ} \mathrm{E}$. In Fig. 2, we see an EB centered at $\sim 13: 20$ UT (indicated by a red arrow in the left top panel). This EB falls into the set of EBs identified in the study by Anagnostopoulos et al. (2010a, b) as precursor signals of the earthquake occurring at 02:46:31 UT on 16 August 2005 near the east coast of Honsu, Japan.

The EB at $\sim 13: 20$ UT on 16 August 2005 was observed during an anodic (night-side) semi-orbit that passed west- ward of the future Japan earthquake epicenter $\left(44.5^{\circ} \mathrm{N}\right.$, $125.6^{\circ} \mathrm{E}$ ); the brown line and the red square on the map of Fig. 2 indicate the segment of the trajectory when the EB was observed by DEMETER and the coordinates where the maximum in the energy band 1 electron intensity was measured. Since the energetic electrons travel along the magnetic field lines, they obviously produce a conjugate EB in the Southern Hemisphere, which is seen with a maximum at $\sim 13: 00$ UT. The position of the conjugate EB appears above Eastern Australia as can be seen by the brown line and the small blue square indicating the part of the same trajectory where the conjugate EB and its intensity maximum was observed by DEMETER.

In the electric field spectrogram in Fig. 2, a strong VLF signal can be seen, which was observed around the time of the earthquake-related EB but for a longer time ( $\sim 6 \mathrm{~min})$. This signal is dispersive and extends in frequencies all over the scale of the panel ranging from 0 to $20 \mathrm{kHz}$. The EB observed above Australia is also related to a broad band, but 

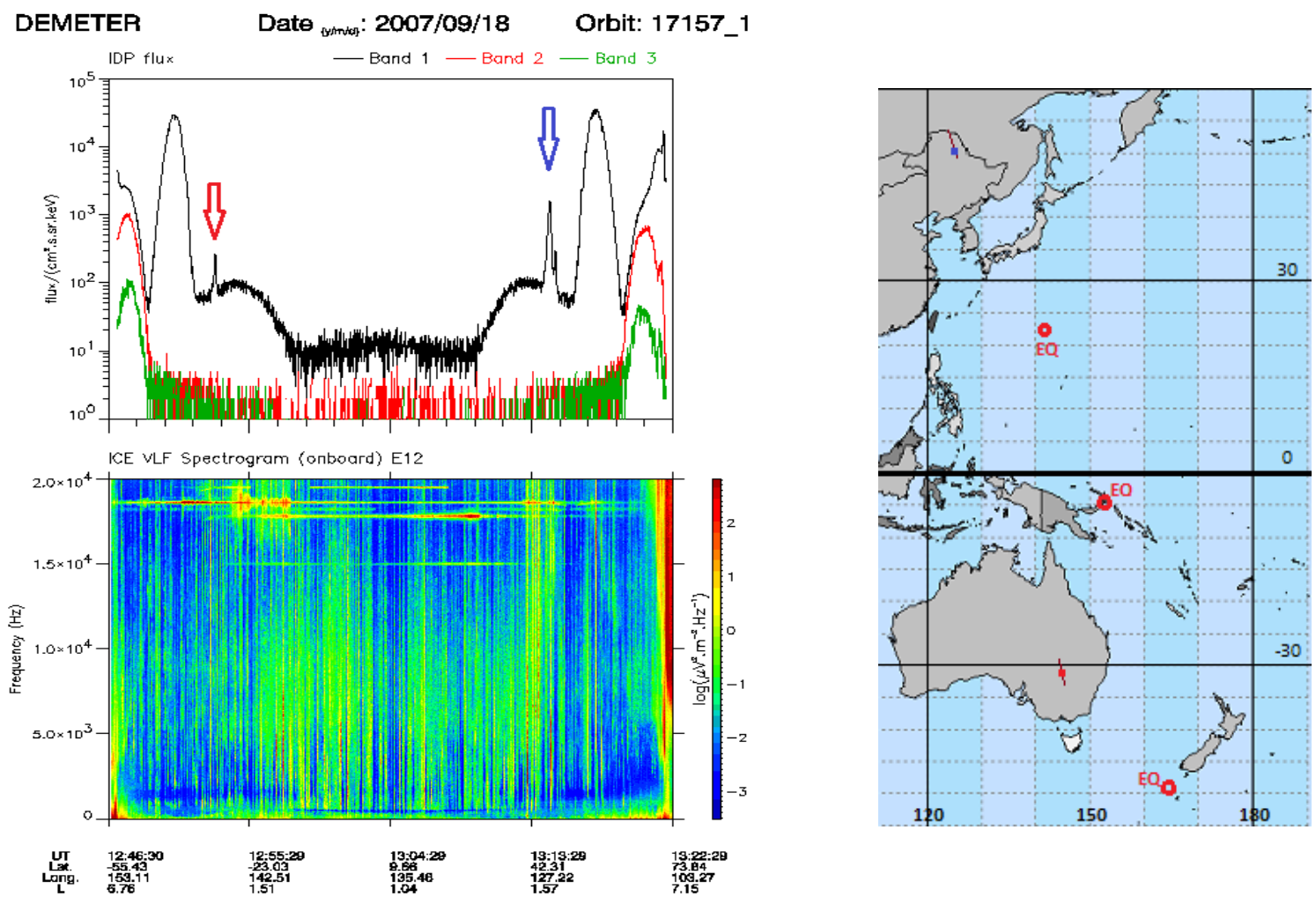

a)

b)

Fig. 3. A clear example of a great earthquake induced electron burst (blue arrow) observed during a non-operational period of the NWC transmitter. Only a characteristic earthquake precursor broad band VLF signal was observed simultaneously with the electron precipitation event (not accompanied by a narrow band emission at $\sim 19.8 \mathrm{kHz}$, since the NWC transmitter was not operating).

weaker in energy and shorter in time $(<1 \mathrm{~min})$ VLF emission, that is self-consistent with the suggestion that this event is the conjugate (not the original) one. We also note that a narrow band signal in the spectrogram of Fig. 2 is also present at the NWC radiating frequency $(19.8 \mathrm{kHz})$, but it is weaker than the signal seen in Fig. 1. We think that the time coincidence of the occurrence of the EB with the intense broad band VLF emission confirms that the earthquake in Japan plays, in this case, the main role in producing the electron precipitation at $\sim$ 13:20 UT on 16 August 2005 (Anagnostopoulos et al., 2010a, b), as we mentioned above.

Since the presence of the most powerful earth-based NWC transmitter in Australia could be considered as the major source of energetic electron events observed eastward of this transmitter (Sauvaud et al., 2008), we analyzed DEMETER observations in this area for a period of several days when NWC was not operating, and obviously, other sources should be considered as agents similar to the NWC-related EBs. Such observations are presented in Fig. 3.

Figure 3 has also been constructed in the same format as Fig. 1, but for the anodic semi-orbit \#17157_1, during the time interval 12:46-13:22 UT, on 18 September 2007. Dur- ing this period, DEMETER crossed the region with longitudes between $127.22^{\circ}-153.11^{\circ} \mathrm{E}$ and a strong anomalous EB was detected at northern latitudes with a maximum flux (peak-to-background ratio $R_{p / b} \approx 20$ ) at $\sim 13: 14: 30 \mathrm{UT}$ (blue square on the map on the right side of Fig. 2). A weaker EB was observed in the Southern Hemisphere.

In the days after the two EBs seen in Fig. 3, significant seismic activity was recorded with three great $(M=$ $6.8,7.5,7.4)$ EQs occurring in a region within $\sim 12^{\circ}$ in longitude, between $142.6^{\circ}-164.12^{\circ} \mathrm{E}$ : (A) $4.99^{\circ} \mathrm{S}, 153.5^{\circ} \mathrm{E} ; M=$ 6.8 , September 26 , (B) $22^{\circ} \mathrm{N}, 142.6^{\circ} \mathrm{E} ; M=7.5$, September 28 , and (C) $49.27^{\circ} \mathrm{S}, 164.12^{\circ} \mathrm{E} ; M=7.4$, September 30 . The EB was observed northward of the epicenters of the three EQs, as usual. We think that the EB observed in the Northern Hemisphere, is probably related to the earthquake occurring at $22^{\circ} \mathrm{N}$ (Mariana Islands, $M=7.5,28$ September), but the presence of other earthquakes at those times might also have contributed to the process.

The EB observed at $\sim 13: 14: 30$ UT on day 18, was accompanied by a broad band emission in the region from a few $\mathrm{kHz}$ to $\sim 20 \mathrm{kHz}$. DEMETER also observed a weaker EB (red arrow) with $R_{p / b} \approx 5$, at southern latitudes (red square 

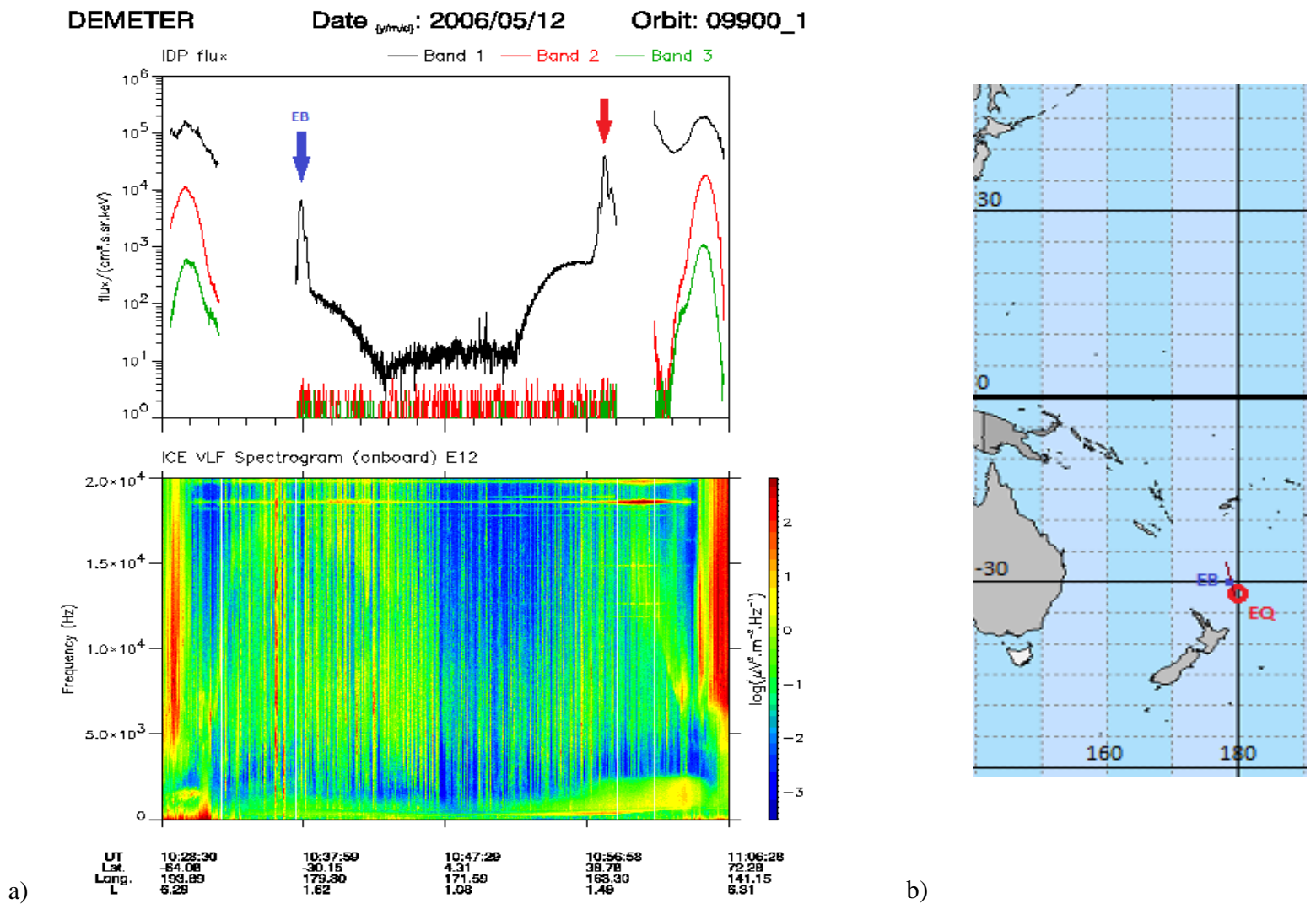

b)

Fig. 4a. Example of an electron burst detected at $\sim 10: 37$ UT on 12 May (blue arrow in the left top panel) observed about four days before the occurrence of a strong earthquake $(M=7.5 R)$ occurring near Kermadec Islands $\left(31.81^{\circ} \mathrm{S}, 180.69^{\circ} \mathrm{E}\right.$; red circle on the map of Fig. $\left.4 \mathrm{a}\right)$ and very close $\left(30.6^{\circ} \mathrm{S}, 179.42^{\circ} \mathrm{E}\right)$ to it. The VLF electric field density presents dispersion in all frequencies with small presence of density concentration at $\sim 19.8 \mathrm{kHz}$ area.

on the map of Fig. 3) during the same trajectory but not accompanied by a special signal in the VLF spectrogram. We consider this small burst as the conjugate one, as opposed to that observed in the Northern Hemisphere. Furthermore, we point out that no narrow band emission was seen in the spectrogram at $\sim 19.8 \mathrm{kHz}$, since the NWC transmitter was not operating. Since the NWC transmitter in Australia was not operating at those times, this implies that the broad band VLF emission related with EBs observed by DEMETER at northern latitudes some days before great earthquakes (eastward of the NWC transmitter) can be identified as an earthquakerelated VLF signal.

Figure 4a has also been constructed in the same format as Fig. 1 and shows an EB detected at $\sim 10: 37$ UT on 12 May (the blue arrow in the top left panel) during DEMETER's anodic semi-orbit \#9900_1. A strong earthquake $(M=7.5 R)$ occurred near Kermadec Islands $\left(31.81^{\circ} \mathrm{S}, 180.69^{\circ} \mathrm{E}\right.$; red circle on the map of Fig. 4 a) very close $\left(30.6^{\circ} \mathrm{S}, 179.42^{\circ} \mathrm{E}\right)$ to the EB of 12 May, about four days later (16 May 2006 at 10:39 UT). The IDP/ DEMETER electron observations we examined for this time period suggest that this EB is included in a set of EBs observed between 1-16 May 2006. These follow the characteristic temporal evolution of earthquake precursor EBs reported by Anagnostopoulos et al. (2010a, b), and show an intensification in the first phase and a reduction of the activity in the second phase which ceases above the epicenter a few hours before the EQ occurrence. This EB presented a strong peak-to-background ratio and was associated with a broad band emission extending in frequency range almost all over the range of the spectrogram, from a few $\mathrm{kHz}$ to at least $20 \mathrm{kHz}$.

The characteristic temporal evolution of electron precipitation for $\sim 15$ days before the Kerdamec Islands EQ (not shown here) and the typical broad band VLF spectral signal suggest that the EB of 12 May is most probably a precursor signal of the 16 May 2006 earthquake.

Figure $4 \mathrm{~b}$ presents DEMETER observations close to the Kerdamec Islands earthquake discussed above, but very close to the time of its occurrence. The measurements shown in Fig. 4b were made in the time interval 09:14:30- 

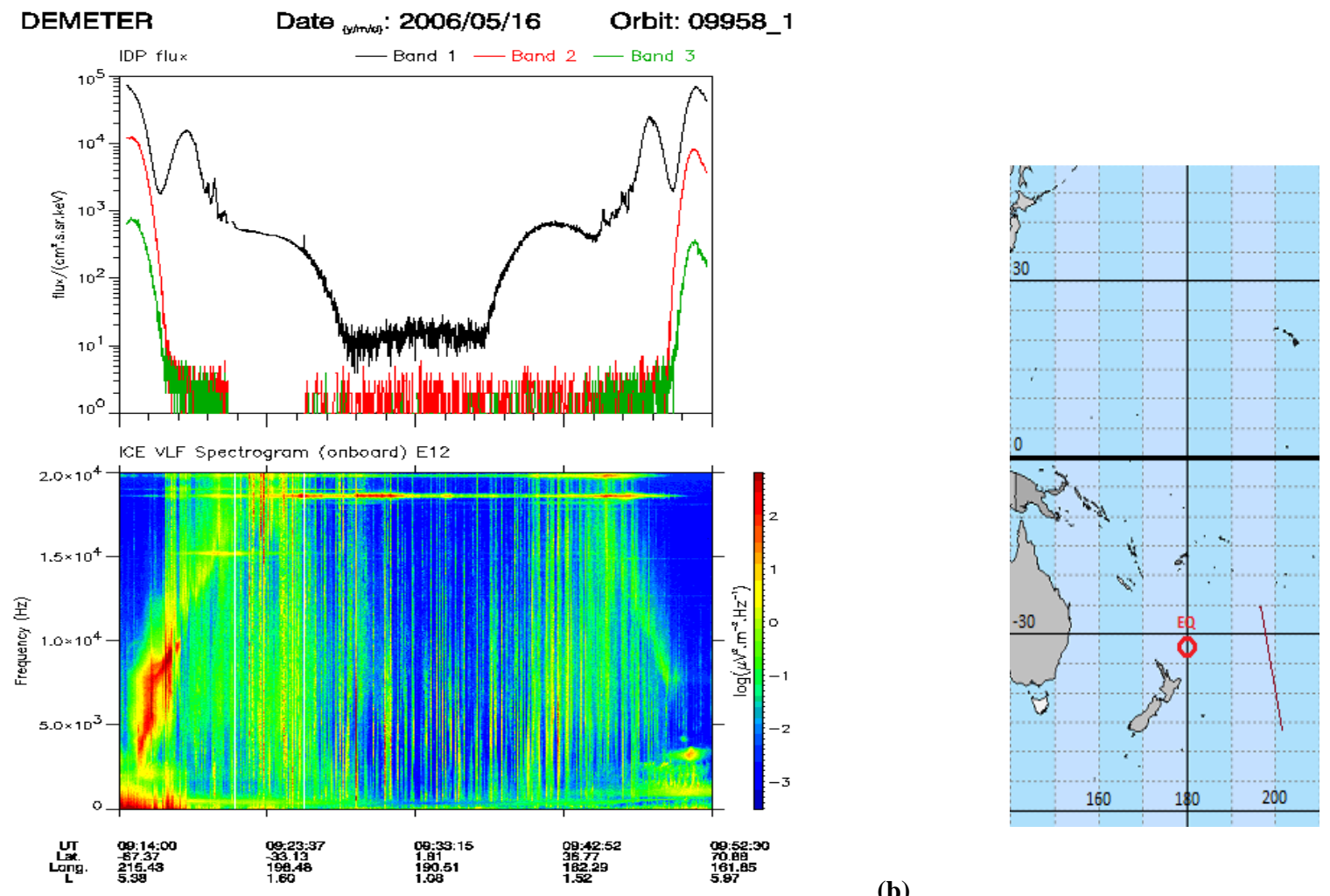

(a)

(b)

Fig. 4b. DEMETER observations close to the great $(M=7.5)$ Kerdamec Islands earthquake shown in Fig. 4a, but very close (about one hour before) to the time of its occurrence. No intense bursts (only small fluctuations) were observed near the epicenter of the earthquake in agreement with the model of Anagnostopoulos et al. (2010a, b), despite the vicinity of the radiating NWC transmitter.

09:52:30 UT, 16 May 2006, which is about one hour before the main earthquake of $M=7.5$, which occurred at 10:39 UT on 16 May 2006 while the spacecraft performed the closest night-side orbit eastward of the future epicenter (map in the right of Fig. 4b). According to the electron precipitation temporal evolution pattern, the electron precipitation activity ceases a few hours before a great earthquake (Anagnostopoulos et al., 2010a). Indeed, Fig. 4b reveals that no intense bursts (only small fluctuations) were observed near the epicenter $\sim 1 \mathrm{~h}$ before the earthquake.

Figure $4 \mathrm{~b}$ shows a similar weak narrow band emission due to the operation of the NWC transmitter, as in the case of Fig. 4a. However, the electron precipitation activity is absolutely different by $\sim 4$ days (Fig. 4 a) and $\sim 1$ h (Fig. 4b) before the strong earthquake of 16 May, eastward and relatively close to the NWC transmitter. Even though the NWC station was continuously radiating, the electron precipitation varied and followed a temporal evolution matching the occurrence time of the strong Kerdamec Islands EQ. These observations suggest that there is no significant influence of the NWC transmitter on radiation belt electrons at $\sim 200^{\circ} \mathrm{E}$ in this case.

\section{Statistical results}

\subsection{Spatial distribution of energetic electron precipitation events}

In order to study the relative contribution of various sources triggering radiation belt electron precipitation events at middle latitudes, we first examined the spatial distribution of DEMETER anomalous energetic electron bursts. The statistical results (to be presented in a paper in preparation) on the strength of DEMETER EBs suggest that a distinct cloud of intense EBs (with high peak-to-background flux ratio) was observed close to the position of the NWC transmitter; such an example of an EB with a flux ratio as high as $R_{p / b}>10^{2}$ was presented in Fig. 1. Based on this finding, we decided to examine the spatial distribution of EBs resembling the kind of NWC-related intense EBs, and we used a relatively high flux threshold $\left(R_{p / b}=20\right)$ for selecting anomalous EBs. Then, we performed a statistical analysis by using a software package for the automatic selection of EBs (detailed information about the whole set of selection EB criteria is given in Appendix B). The spatial distribution of EBs selected in this way is displayed in Fig. 5 for two time intervals, Period I 


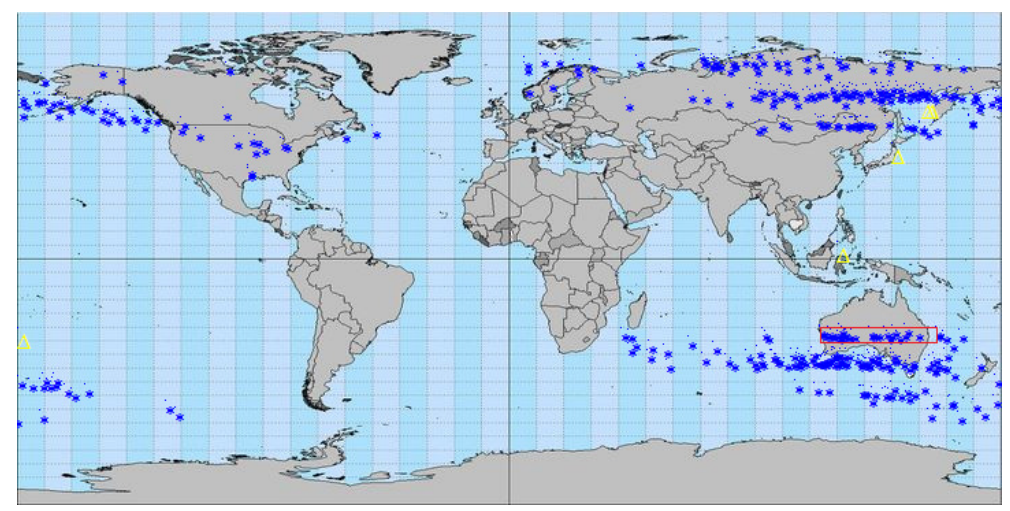

(a)

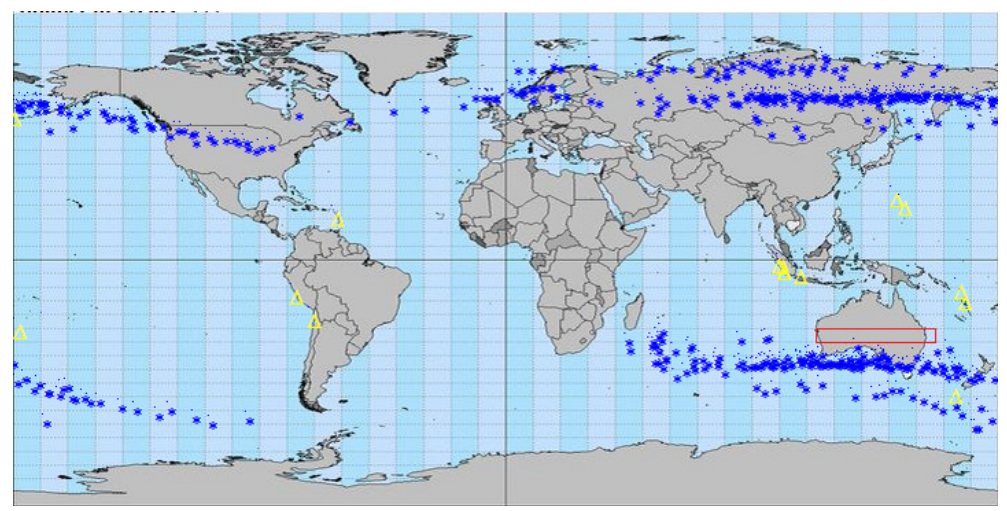

(b)

Fig. 5. DEMETER/IDP intense (peak-to-background flux ratio $R_{p / b}>20$ ) electron bursts during two 6-month periods under conditions when the NWC powerful transmitter in Western Australia was turned on (a) and off (b). A cluster of electron bursts is evident between latitudes $\sim 25^{\circ}-30^{\circ} \mathrm{S}$ and longitudes $\sim 114^{\circ}-167^{\circ} \mathrm{E}$, when the transmitter was operating (area marked with red rectangle).

and Period II. The selection of these two statistical samples was based on the longest time interval, from 1 July 2007 to 22 January 2008 (Period II) when the NWC transmitter was not operating (Gamble et al., 2008). Period I, from 1 July 2008 to 31 December 2008, is used for comparatively studying the EB spatial distribution under the influence of the NWC transmitter. Period I has a similar time interval to that of period II (second half of a year), in order to minimize possible seasonable effects.

In Fig. 5 we display the EB space distribution on the Earth's map for Period I (top panel) and Period II (bottom panel), as resulting from the automatic selection of EBs from our algorithm mentioned above. Here we should point out that our criteria are such that they miss electron precipitation events at low and high latitudes.

The set of intense radiation belt precipitation electron events peak-to-background flux ratio $\left(R_{p / b}>20\right)$ of Fig. 5 displays the general form of the electron spatial distribution known from previous studies (Sauvaud et al., 2008). For instance, in the bottom panel of Fig. 5 we can distinguish the signature of the outer belt electrons in the cluster of asterisks at $\sim 40^{\circ} \mathrm{S}$, between $80^{\circ}-160^{\circ} \mathrm{E}$ and the auroral elec- trons as a cluster at higher latitudes. A large area around the South Atlantic Anomaly $\left(\sim 80^{\circ} \mathrm{W}-40^{\circ} \mathrm{E}\right)$ is devoid of points, since our algorithm selects events on the basis of intense peak-to-background flux ratio, not on the basis of absolute fluxes. In the top panel, during Period I, an additional set of points can be seen above the outer radiation belt electron points, between latitudes $\sim 25^{\circ}-30^{\circ} \mathrm{S}$, and longitudes $\sim 114^{\circ}-167^{\circ} \mathrm{E}$, which is obviously related to the NWC transmitter activity; this is implied from the fact that this electron event set was only observed during Period I, when the NWC transmitter was operating (NWC on) and was not observed during Period II, when the transmitter was turned off, as well as from the vicinity of these events with regard to the position of the transmitter. Other powerful earth- based transmitters are also operating in various countries around the world. Green and Fung (2005) reported that they identified over 100 stations generating emissions in the frequency range $10-50 \mathrm{kHz}$ ). Therefore, since a separate cluster of EBs can be seen in the north of Europe around Scandinavia (more evident during Period II; bottom panel of Fig. 5), it is possible that this is a result of numerous transmitters located in Europe. 

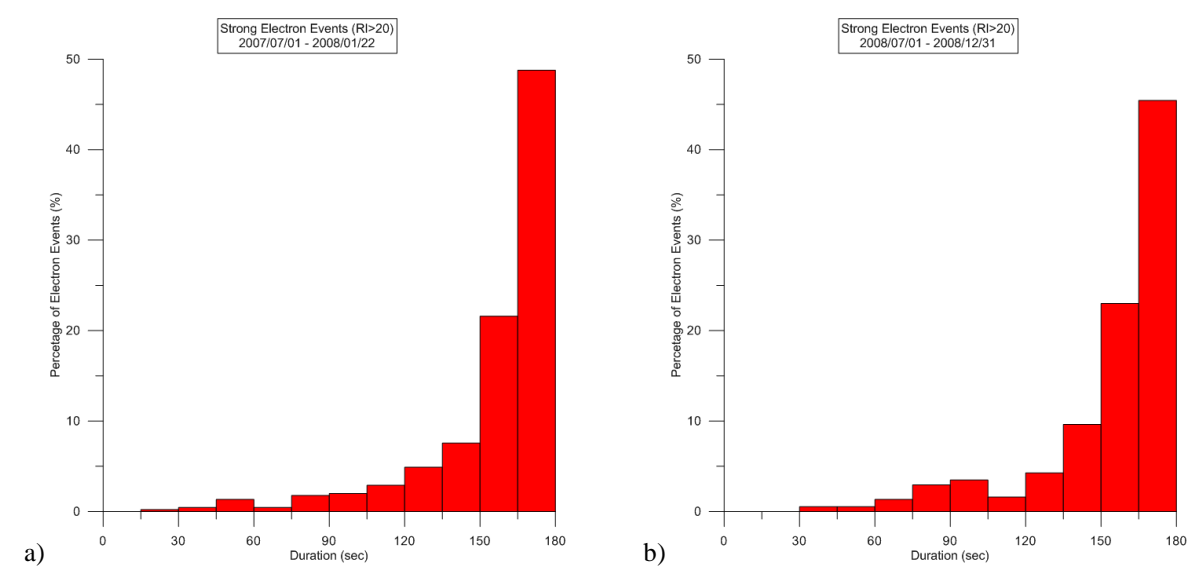

Fig. 6. Time duration distributions for the strong EB events recorded (a) during a NWC non-operational period and (b) during an NWC operational period. On both distributions over $\sim 80 \%$ of the recorded EB events had a duration of over $120 \mathrm{~s}$, giving a clear distinction from events originated by lighting, which have a very small duration of a few seconds.

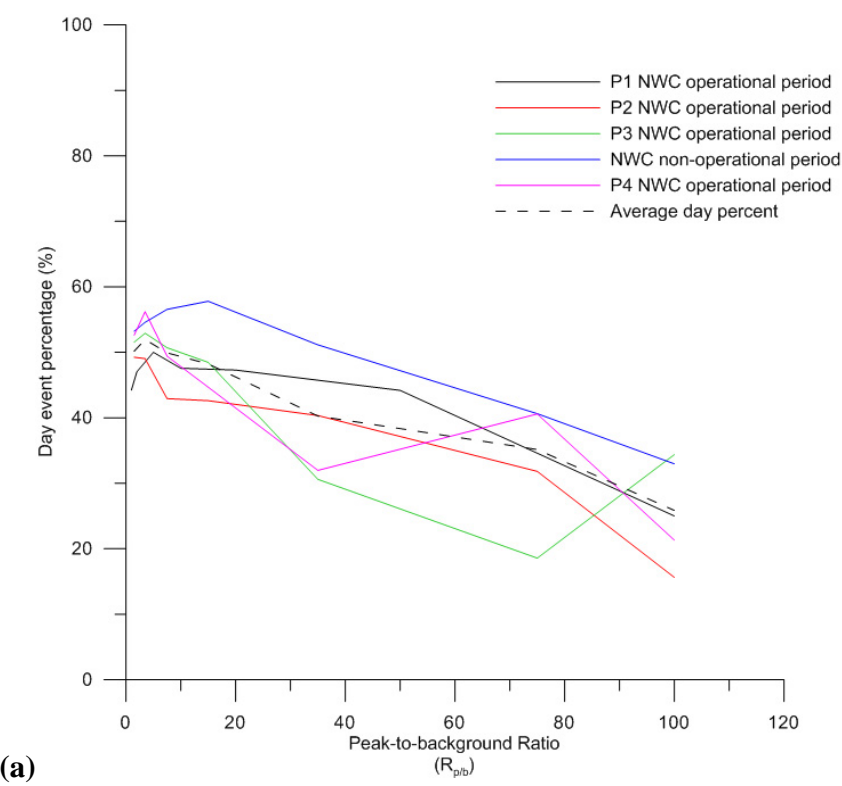

(b)

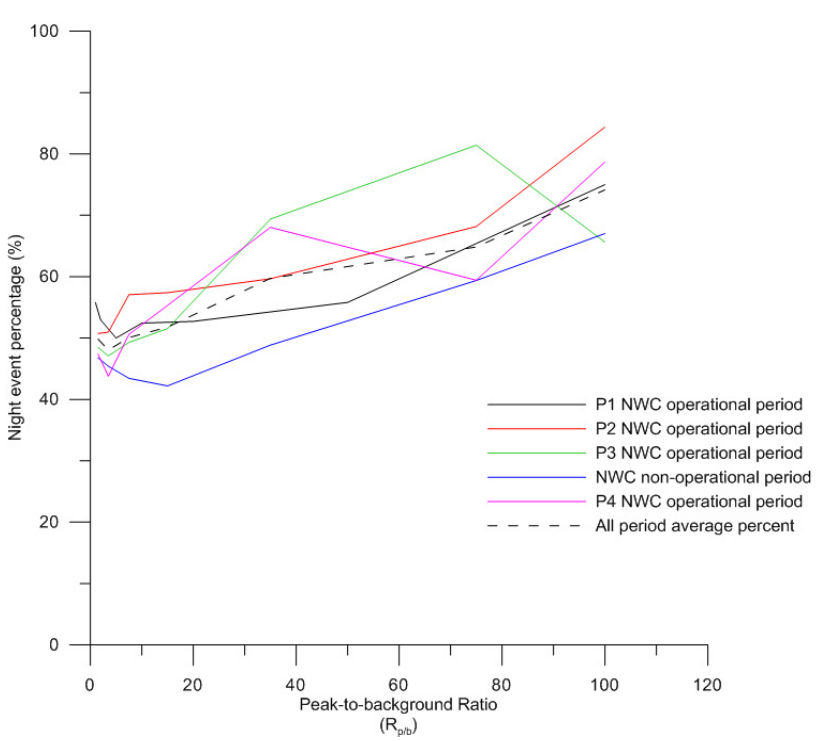

Fig. 7. Percentage of the EBs as a function of their peak-to-background value $R_{p / b}$ for 06:00-18:00 LT (panel a) and 18:00-06:00 LT (panel b). The comparison of the two distributions obviously suggests a day-night asymmetry in the detection of the DEMETER electron bursts, with a optimum presence of more strength electron events in the night-side plasmasphere. However, it is remarkable that when the NWC transmitter was turned off, the inclination of the line shows it is the smallest of all other lines in both distributions (Fig. 7a, b).

Finally, we note that the EB distributions seen in Fig. 5 suggest that strong EBs cover the space both above ground and sea.

Table 1 summarizes some interesting numbers for the two statistical samples of Fig. 5; Table 1 shows, for Period I and Period II, the global number of great $(M>7)$ earthquakes, the global number of EBs and of the cluster of the NWCassociated EBs. It also shows the occurrence frequency of all EBs observed by DEMETER and selected by our algorithm when the restriction for high values (peak-to-background flux ratio $R_{p / b}>10^{2}$ ) was not applied. We see that: (a) a higher number of EBs was observed globally during Period II, when more earthquakes were recorded and the powerful NWC transmitter was not broadcasting than during Period I with lesser intense earthquakes while the NWC transmitter was operating (449 EBs during Period II with 14 EQs versus 374 EBs during period I with 5 EQs), (b), no EBs (from a total of 449 EBs) were observed in the same area when the transmitter was off, (c) intense EB in the NWC influence area were observed at a low occurrence frequency $(2.1 \%)$ 
Table 1. Statistical analysis for the two 6-month periods.

\begin{tabular}{lll}
\hline & Period I & Period II \\
\hline NWC transmitter & ON & OFF \\
Earthquakes $(>7)$ & 5 & 14 \\
Intense $\left(R_{p} / b>20\right)$ events & 374 & 449 \\
Intense NWC influenced electron events & 17 & 0 \\
Percentage of orbits with intense NWC-influenced area events & $2.1 \%$ & \\
Percentage of orbits with NWC-influenced area bursts & $9.3 \%$ & \\
Percentage of NWC-influenced events (globally) & $\sim 1.5 \%$ & \\
\hline
\end{tabular}

of DEMETER passes during Period I, and (d) 34 intense NWC-associated EB's (including the cluster of points seen in the Northern Hemisphere in the region between $110^{\circ}-150^{\circ} \mathrm{E}$ and $46^{\circ}-49^{\circ} \mathrm{N}$, considered to be the conjugated EBs) were observed compared to a total of 2221 EBs recorded globally during Period I, which represents a percentage as low as $\sim 1.5 \%$.

\subsection{Time duration of energetic electron precipitation events}

We mentioned above in the description of Fig. 5 that strong EBs cover the space both above ground and sea. Since lighting is observed only above ground, it is rather impossible that lighting can be a significant source of the EB sample examined in Fig. 5, at least above the sea, where a significant percentage of EB's was detected. In order to further check the possible contribution of lighting in generating strong EBs like those observed near the NWC transmitter and in general included in the statistical samples I and II (Fig. 5), we examined the time duration of the DEMETER electron events shown in Fig. 5 and we present them with histograms in Fig. 6. The results shown in Fig. 6, for both Period I and II, imply that a percentage $\mathrm{P}>\sim 80 \%$ of the EBs lasted for more than $2 \mathrm{~min}$ and that no EB lasted for $<15 \mathrm{~s}$. Such long duration times of the EBs suggest that the EBs examined here cannot be attributed to lighting, since the duration times of energetic electron spikes triggered by lighting are of the order of a few seconds (Chang and Inan, 1983).

\subsection{Day-night asymmetry}

A day-night asymmetry is known to exist in VLF waves trapped in the plasmasphere. Since radiation belt electron precipitation is due to VLF wave-electron interaction, we wanted to check if such an asymmetry is present in EEP events. For this reason, we evaluated and present in Fig. 7 the percentage $\mathrm{P}$ of the EBs as a function of their strength (indicated by their peak-to-background value $R_{p / b}$ ) for 06:0018:00 LT (panel a) and 18:00-06:00 LT (panel b).

In Fig. 7 we included results for the period when the NWC transmitter was turned off (1 July 2007-22 January 2008) and four other similar $\sim 6$ month time intervals: P1 (11 August 2004-22 January 2005), P2 (1 July 2005-22 January 2006), P3 (1 July 2006-22 January 2007) and P4 (1 July 2008-31 December 2008; there is a gap in our analysis between 1 January 2009-11 January 2009 because of a problem in processing the IDP data during that period). The dashed line in the figure corresponds to the average percentage over all five periods investigated.

From Fig. 7 we see that in the dayside plasmasphere P increases almost monotically as $R_{p / b}$ decreases whereas in the nightside plasmasphere, it does so as $R_{p / b}$ increases. The comparison of the two distributions obviously suggests a day-night asymmetry in the detection of the DEMETER EBs, with an optimum presence of more strength electron events in the night-side plasmasphere.

Another interesting point to note from Fig. 7 is that for the time interval when the NWC transmitter was turned off, the inclination of the line appears low and smallest of all other lines in both distributions (Fig. 7a, b). This observational finding suggests that the decreased anthropogenic VLF emission due to the non-operation of a powerful ground-based transmitter resulted in a remarkable weakness of the daynight asymmetry. This finding may suggest some difference in the generation process of anthropogenic and naturallycaused electron precipitation that should be studied in the future.

\section{Summary of observations and conclusions}

In this study we have attempted to estimate, for the first time, the partial contribution of various natural and anthropogenic sources in the generation of radiation belt electron precipitation events at middle latitudes $\left(>\sim 23^{\circ} \mathrm{N} / \mathrm{S}\right)$ by a satellite in polar orbit (DEMETER). The relatively long time duration of events (and the detection over the sea) studied here excludes the contribution of lightning-generated (short lived) events from our analysis and, therefore, allows the direct comparison of the contribution of the earthquake- and ground-based transmitter-caused electron precipitation events.

Our comparative study of DEMETER electron observations with electric field spectrograms suggests that the 
electron precipitation events triggered by seismic activity are accompanied by broadband $\mathrm{kHz}$ emissions $(\sim 1-20 \mathrm{kHz})$, whereas the ground-based transmitter-related events are normally accompanied by a narrow frequency band emission, centered at the emitting frequency (for instance around $19.8 \mathrm{kHz}$ in the case of the transmitter of US Navy in Western Australia).

In order to estimate the relative contribution of the two sources, the great earthquakes and the ground-based transmitters, we used the statistical analysis of electron burst characteristics during two $\sim 6$ month periods as a tool. The main results of our statistical analysis are the following: (1) the NWC transmitter produced much stronger electron precipitation effects than all other transmitters, (2) intense EBs were observed at a percentage of $\sim 2.1 \%$ of the DEMETER passes above the NWC transmitter influence area $\left(25^{\circ}-30^{\circ} \mathrm{S}\right.$, $114^{\circ}-167^{\circ} \mathrm{E}$ ) in the time period examined, (3) the percentage of NWC-associated EBs (including the NWC conjugate EBs in the Northern Hemisphere) was only $\sim 1.5 \%$ of the whole number of EBs, (4) more EBs were observed when the most powerful ground-based transmitter was off (Period II) than during a similar $\sim 6$ month time interval (Period I) when the transmitter was operating (449 versus $374 \mathrm{EBs}$ in the two cases), (5) during the Period II a higher number of great earthquakes occurred than in Period I (14 versus 5), (6) more intense EBs were observed in the night side than in the day side, with the day-night asymmetry found to be much weaker for the period when the powerful NWC transmitter was closed.

The above Points \# 1-3 confirm the results of previous published papers on the major influence of the NWC transmitter on the inner radiation belts among all other transmitters (Gamble et al., 2008; Sauvaud et al., 2008), but its total contribution to the electron precipitation events all over the globe appears to be very low. Point \# 1 and 4 strongly suggest that earthquakes, and not VLF transmitters, are the major agent of the EBs analyzed in the present study.

It is generally accepted that an increase in the concentration of VLF waves leads to an increase in the interaction with electrons of the Van Allen radiation belts and that cyclotron resonance interaction between VLF waves and electrons leads to variations in the phase space of electron velocity (Pulinets and Boyarchuk, 2004). Consequently, some energetic electrons obtain pitch angle in the loss cone, and penetrate into the ionosphere (Inan et al., 1978). However, Point \#5 suggests some difference in the generation process of anthropogenic and naturally-caused electron precipitation, but this differentiation should be further checked.

The results of the present study support the proposal that the detection of radiation belt electron precipitation is a useful tool for earthquake prediction research (Anagnostopoulos et al., 2010a). In a paper under preparation, we will present an even more detailed comparative study on the relative contribution of earthquake- and the anthropogenic-related electron precipitation events.

\section{Appendix A}

To quantify the strength of the electron burst we used a quantity called "Peak-to-Background Ratio" $R_{p / b}$ :

$$
R_{p / b}=\frac{f_{\max }}{f_{\mathrm{bg}}}
$$

where $f_{\max }$ is the maximum intensity of the electron burst and $f_{\mathrm{bg}}$ the "background" intensity at the time of $f_{\max }$, where the value taken from a linear interpolation between the starting and the ending point (intensity) of the burst is defined as "background" intensity.

The above quantities are written as follows:

$$
f_{\text {bg }}=f_{\text {start }}+A\left(t_{\text {max }}-t_{\text {start }}\right), \quad A=\frac{f_{\text {end }}-f_{\text {start }}}{t_{\text {end }}-t_{\text {start }}}
$$

So Eq. (A1) leads to:

$$
R_{p / b}=\frac{f_{\max }}{f_{\text {start }+\mathrm{A}\left(\mathrm{t}_{\max }-\mathrm{t}_{\text {start }}\right)}}
$$

\section{Appendix B}

For identification of an energetic electron intensity enhancement as a burst we required:

1. A statistically significant $>40 \%$ increase in the intensity of electrons in the energy range $72-526 \mathrm{keV}$ (defined as Band 1 in the text) within the readouts of the "survey mode" ( $4 \mathrm{~s})$ of the IDP instrument, that is

$$
\frac{j[i+1]}{j[i]}>1.4
$$

where $\mathrm{j}[\mathrm{i}]$ and $\mathrm{j}[\mathrm{i}+1]$ are two ("survey mode") successive values of electron intensity. (when DEMETER was operating in the "burst mode" ( $1 \mathrm{~s})$, the intensity was averaged over $4 \mathrm{~s}$ ).

1. An increase from an intensity $j[i]$ to $j[i+1]$ was defined as statistically significant if

$j[i+1]-\lambda \sigma(j[i+1])>j[i]+\lambda \sigma(j[i])$

where we put $\lambda=1.1$

1. Remarkable intensity increases, with a $R_{p / b}>2$ were only included in our statistical sample.

2. A maximum duration of the electron event was set at $180 \mathrm{~s}$ (with a maximum in the intensity of the event required to be met within $2 / 3$ of the total duration).

3. When an electron followed a similar increase event intensity enhancement in energy Band $2(526-971 \mathrm{keV})$ as in energy Band 1 (citeria a and b), it was excluded from the statistical sample. 
Acknowledgements. The authors thank Sauvaud and Berthelier for providing the DEMETER data. We also thank the post-graduate student E. Vassiliadis for his help in data processing.

Edited by: K. Eftaxias

Reviewed by: two anonymous referees

\section{References}

Abel, B. and Thorne, R. M.: Electron scattering and loss in Earth's inner magnetosphere: 1. Dominant physical processes, J. Geophys. Res., 103, 2385-2396, 1998.

Aleksandrin, S. Yu., Galper, A. M., Grishantzeva, L. A., Koldashov, S. V., Maslennikov, L. V., Murashov, A. M., Picozza, P., Sgrigna, V., and Voronov, S. A.: High-energy charged particle bursts in the near-Earth space as earthquake precursors, Ann. Geophys., 21, 597-602, doi:10.5194/angeo-21-597-2003, 2003.

Anagnostopoulos, G., Rigas, V., Athanasiou, M., Iliopoulos, A., Vassiliadis, E., and Iossifidis, N.: Temporal Evolution of Energetic Electron Precipitation as a promising tool for Earthquake Prediction Research: Analysis of IDP/DEMETER Observations, in: Advances in Hellenic Astronomy during the IYA09, ASP Conference Series, edited by: Tsinganos K., Hatzidimitriou D., Matsakos T., 424, 67-74, 2010a.

Anagnostopoulos, G., Rigas, V., and Vassiliadis, E.: Radiation belt electron precipitation in the upper ionosphere at middle latitudes before strong earthquakes, (http://xxx.lanl.gov/abs/1012.3588), 2010b.

Anagnostopoulos, G., Papandreou, A., and Antoniou, P.: Solar wind triggering of geomagnetic disturbances and strong $(M>$ 6.8) earthquakes during the November-December 2004 period, http://xxx.lanl.gov/abs/1012.3585, 2010c.

Athanasiou, M. A., Anagnostopoulos, G. C., Iliopoulos, A. C., Pavlos, G. P., and David, C. N.: Enhanced ULF radiation observed by DEMETER two months around the strong 2010 Haiti earthquake, Nat. Hazards Earth Syst. Sci., 11, 1091-1098, doi:10.5194/nhess-11-1091-2011, 2011.

Berthelier, J. J., Godefroy, M., Leblanc, F., Malingre, M., Menvielle, M., Lagoutte, D., Brochot, J. Y., Colin, F., Elie, F., Legendre, C., Zamora, P., Benoist, D., Chapuis, Y., Artru, J., and Pfaff, R.: ICE, The electric field experiment on DEMETER, Planet. Space Sci., 54, 5, 456-471, 2006.

Bošková, J., Šmilauer, J., Tříska, P., and Kudela, K.: Anomalous behaviour of plasma parameters as observed by the Intercosmos 24 satellite prior to the Iranian earthquake of 20 June 1990, Stud. Geophys. Geod., 38, 2, 213-220, 1994.

Chang, H. C. and Inan, U. S.: Lighting-induced electron precipitation from the Magnetosphere, J. Geophys. Res., 90, A2, 15311541, 1983.

Contoyiannis, Y. F., Kapiris, P. G., and Eftaxias, K. A.: Monitoring of a preseismic phase from its electromagnetic precursors, Phys. Rev. E., 71, 6, 066123-1-061123-14, 2005.

Fidani, C. and Battiston, R.: Analysis of NOAA particle data and correlations to seismic activity, Nat. Hazards Earth Syst. Sci., 8, 1277-1291, doi:10.5194/nhess-8-1277-2008, 2008.

Galper, A. M., Grachev, V. M., Dmitrienko, V. V., KirillovUgryumov, V. G., Polukhina, N. G., Tzarkov, R. N., and Ulin, S. E.: Spatial-temporal correlation of the earthquakes and varia- tions of high energy flux in the inner radiation belt, Cosmic Res., 21, 707-717, 1983

Gamble, R. J., Rodger, C. J., Cliverd, M. A., Sauvaud, J.-A., Thomson, N. R., Stewart, S. L., Mccormick, R. J., Parrot, M., and Berthelier, J.-J.: Radiation belt electron precipitation by man-made VLF transmissions, J. Geophys. Res., 113, A10211, doi:10.1029/2008JA013369, 2008.

Ginzburg, E. A., Malishev, A. B., Proshkina I. P., and Pustovetov, V. P.: Correlation of strong earthquakes with radiation belt particle flux variations, Geomagn Aeronomy+, 34, 315-320, 1994.

Graf, K. L., Inan, U. S., Piddyachiy, D., Kulkarni, P., Parrot, M., and Sauvaud J. A.: DEMETER observations of transmitter-induced precipitation of inner radiation belt electrons, J. Geophys. Res., 114, A07205, doi:10.1029/2008JA013949, 2009.

Green L. and Fung, S. F.: Advances in Inner Magnetosphere Passive and Active Research, in: Inner Magnetosphere Physics and Modeling, edited by: Pulkkinen, T., Tsyganenko, N. and Friedel, R., AGU Geoph. Monograph, 155, 181, 2005.

Hayakawa, M. R., Kawate, R., and Molchanov, O. A.: Ultra-low frequency signatures of the Guam earthquake on 8 August 1993 and their implication, J. Atmos. Elec., 16, 3, 193-198, 1996.

Inan, U., Bell, T., and Helliwell, R.: Nonlinear Pitch Angle Scattering of Energetic Electrons by Coherent VLF Waves in the Magnetosphere, J. Geophys. Res., 83, A7, 03235, doi:10.1029/JA083iA07p03235, 1978.

Katoh, Y., Takayuki, O., and Masahide, I.: A Numerical Study on the Resonant Scattering Process of Relativistic Electrons via Whistler-Mode Waves in the Outer Radiation Belt, in: Inner Magnetosphere-Physics and Modeling, edited by: T. Pulkkinen, N. Tsyganenko and R Friedel, AGU Geoph. Monograph, 155, 33, 2005.

Koskinen, H.: Energetic Particles losses from the Inner Magnetosphere, in Inner Magnetosphere-Physics and Modeling, edited by: Pulkkinen, T., Tsyganenko, N. and Friedel, R., AGU Geoph. Monograph, 155, 23, 2005

McCormick, R. J., Rodger, C. J., and Thomson, N. R.: Reconsidering the effectiveness of quasi-static thunderstorm electric fields for whistler duct formation, J. Geophys. Res., 107, 1396, doi:10.1029/2001JA009219, 2002.

Nmec, F., Santolík, O., and Parrot, M.: Decrease of intensity of ELF/VLF waves observed in the upper ionosphere close to earthquakes: A statistical study, J. Geophys. Res., 114, A04303, doi:10.1029/2008JA0139, 72, 2009.

Paulikas, G. A.: Precipitation of particles at Low and Middle Latitudes, Rev. Geophys. Space Ge., 13, 5, 709, 1975.

Pulinets, S. and Boyarchuk, K.: Ionospheric Precursors of Earthquakes, Springer, ISBN3-540-20839-9, 2004.

Rothkaehl, H., Bucik, R., and Kudela K.: Ionospheric plasma response to the seismic activity, Phys. Chem. Earth, 31, 4-9, 473481, 2006.

Sauvaud, J. A., Moreau, T., Maggiolo, R., Treilhou, J.-P., Jacquey, C., Cros, A., Coutelier, J., Rouzaud, J., Penou, E., and Gangloff, M.: High-energy electron detection onboard DEMETER: The IDP spectrometer, description and first results on the inner belt, Planet. Space. Sci., 502-511, 2006.

Sauvaud, J.-A, Maggiolo, R., Jacquey, C., Parrot, M. Berthelier, J.-J., Gamble, R. J., and Rodger, C. J.: Radiation belt electron precipitation due to VLF transmitters: Satellite observations'. Geophys. Res. Lett., 35, L09101, doi:10.1029/2008GL033194, 
2008., 2008.

Sgrigna, V., Carota, L., Conti, L., Corsi, M., Galper, A. M., Koldashov, S. V., Murashov, A. M., Picozza, P., Scrimaglio, R., and Stagni, L.: Correlations between earthquakes and anomalous particle bursts from SAMPEX/PET satellite observations, J. Atmos. Sol.-Terr. Phy., 67, 15, 1448-1462, 2005.

Sidiropoulos, N., Anagnostopoulos, G., and Rigas, V.: Radiation belt electron precipitation in the upper Ionosphere $(700 \mathrm{~km})$ : Earthquake induced or ground transmitter stimulated?, Proc. of 15th Conference Microwave Techniques COMITE 2010, Brno, Czech Rep., 19-21 April, 161-164, 2010.
Vampola, A. L. and Gorney, D. J.: Electron energy deposition in the middle atmosphere, J. Geophys. Res, 88, 6267-6274, 1983.

Zhang, X., Shen, X., Parrot, M., Zeren, Z., Ouyang, X., Liu, J., Qian, J., Zhao, S., and Miao, Y.: Phenomena of electrostatic perturbations before strong earthquakes on DEMETER, Nat. Hazards Earth Syst. Sci., in review, 2011. 